cannot perfectly reflect the incidence of EPS. Owing to the limitation of our dataset (which did not include indications for prescriptions), we cannot exclude the possibility that some patients may have been prescribed antiparkinsonian medication because they had Parkinson's disease, not because they had EPS caused by antipsychotics.

Grover \& Kulhara question why we included only 266 GPs in this study. We selected from the GPRD only those patients who had been diagnosed with schizophrenia and prescribed antipsychotics between 1992 and 2000. Therefore 6356 patients who met those requirements and their 266 general practices were included in the study.

Grover \& Kulhara raise the possibility that patients might have taken both classes of antipsychotics simultaneously. We examined the effects of switching antipsychotics on antiparkinsonian drug prescribing by classifying patients into two groups. We defined the TA group as patients who had been prescribed typical antipsychotics with no atypical antipsychotic use before the switch, completely stopped typical antipsychotics and subsequently switched to atypical antipsychotics, with no typical antipsychotic use for at least 2 years after the switch. The TT group included patients who were prescribed one typical antipsychotic (e.g. chlorpromazine) then switched to a different typical antipsychotic (e.g. haloperidol), and who never received an atypical antipsychotic during the study period. Therefore, by definition, no patients in our study were receiving a combination of both classes of antipsychotics.

Barak, Y., Shamir, E. \& Weizman, R. (2002) Would a switch from typical antipsychotics to risperidone be beneficial for elderly schizophrenic patients? A naturalistic, long-term, retrospective, comparative study. Journal of Clinical psychopharmacology, 22, II5-120.

Bobes, J., Gilbert, J., Ciudad, A., et al (2003) Safety and effectiveness of olanzapine versus conventional antipsychotics in the acute treatment of first-episode schizophrenic inpatients. Progress in Neuro-Psychopharmacology and Biological Psychiatry, 27, 473-48I.

Montes, J. M., Ciudad, A., Gascon, J., et al (2003) Safety, effectiveness, and quality of life of olanzapine in first-episode schizophrenia: a naturalistic study. Progress in Neuro-Psychopharmacology and Biological Psychiatry, 27, 667-674.

S. Park Korea Health Industry Development Institute, 57-I Noryangjin-Dong, Dongjak-GU, Seoul 156-800, Republic of Korea.

E-mail: sylviap@khidi.or.kr
D. Ross-Degnan, A. S. Adams,

J. Sabin Department of Ambulatory Care and Prevention, Harvard Medical School and Harvard Pilgrim Health Care, Boston, USA

P. Kanavos London School of Economics and Political Science, London, UK

S. B. Soumerai Department of Ambulatory Care and Prevention, Harvard Medical School and Harvard Pilgrim Health Care, Boston, USA

\section{Treatment of borderline personality disorder}

Fonagy \& Bateman (2006) hypothesise that a more benign course of borderline personality disorder may partially result from a reduction in iatrogenic harm. They describe people with borderline personality disorder as having 'hyperactive attachment systems' which interfere with the therapeutic relationship and treatment. They describe 'treatment' as being psychosocial treatment or psychotherapy, and attachment figures as therapists.

Many people with borderline personality disorder do not receive psychotherapy but do have contact with psychiatric services - casualty assessments, out-patient contact with generic services, brief crisis admissions and sometimes even prolonged admissions. I am curious as to Fonagy $\&$ Bateman's view on the nature of attachments that people with borderline personality disorder have with psychiatric institutions, especially when contact with individual workers may be inconsistent. Fonagy \& Batemen give advice about how to encourage 'mentalisation' in the context of psychotherapy in order to avoid potential iatrogenic damage but give no advice for other clinical settings.

Clinical teams are well aware of how people with borderline personality disorder may unconsciously 'engineer' situations to re-enact disturbed early life experiences. Now Fonagy \& Bateman suggest that although teams are aware of this situation further damage may be done. A 'helpful' intervention may deprive the patient of using or developing other more useful strategies. Fonagy \& Bateman suggest that an 'inquisitive and flexible' approach may be useful. The challenge is therefore how this approach should be applied to how clinical teams within institutions respond to people with borderline personality disorder.
Fonagy, P. \& Bateman, A. (2006) Progress in the treatment of borderline personality disorder. British Journal of Psychiatry, 188, I-3.

D. Mountain Royal Edinburgh Hospital, Morningside Terrace, Edinburgh EHIO 5HJ, Scotland. E-mail: debbie.mountain@lpct.scot.nhs.uk

Authors' reply: We share Dr Mountain's concern that this group of patients is often inadequately managed. Our primary aim in pointing to the iatrogenic consequences of psychotherapy was to illustrate the dangers of intensive interventions or those with poorly defined boundaries. The same concerns for iatrogenic consequences apply to institutional involvement because this is often disrupted by frequent staff changes. Separations and losses of this kind are also iatrogenic. They activate patients' attachment systems, leading them to make unproductive attempts to restabilise their sense of self. Moreover, interactions with institutions often occur at times of personal crisis when the attachment system is already stimulated. Concerns about the patient's state of panic and about reduced mentalising may lead to hospital admission. However, this can become iatrogenic in itself because emotionally charged interactions with staff and other patients may further destabilise the patient, leading them to self-harm or threaten suicide, prolonging hospital admission. We and others (Paris, 2004) recommend that the level of risk for self-harm of patients admitted to hospital should be assessed and documented daily. If there is no reduction in risk, alternative management of the patient in the community should be implemented.

Although patients may seem to be enacting past experiences in their interactions with clinical teams, in our view it is not useful to consider these as hapless repetition of past patterns or as acts that respond to or compensate for past hurts; rather they should be viewed as the only solution available to restore a sense of integrity, continuity and coherence. The provision of a highly integrated model of psychiatric care in a structured institutional environment that aims to offer consistent, coherent and thoughtful psychological care with a relationship focus, organised in a patient-oriented flexible manner with individualised care plans, is likely to be most helpful. Out-patient treatment, discharge from an in-patient unit or referral following a casualty visit should be considered in 